\title{
Agenesia de rama derecha de la arteria pulmonar
}

\author{
Agenesis of right pulmonary artery \\ Mario Soto-Ramos, ${ }^{*, \neq \S}$ Raúl Hernández-Saldaña, ${ }^{*, \neq, \S}$ Luis Carlos Hinojos-Gallardo, ${ }^{*, \neq, \mathbb{}}$ \\ Miguel Alejandro Mendoza-Enriquez, ${ }^{* \neq}$ Yazmín Alejandra Márquez-Rodríguez, ${ }^{*, \neq}$ Noé Guerrero-Lucio, \\ *Universidad Autónoma de Chihuahua; ${ }^{\ddagger}$ Hospital Infantil de Especialidades de Chihuahua; § Hospital Ángeles Chihuahua; \\ "Hospital Star Médica. Chihuahua, México.
}

\begin{abstract}
RESUMEN. Dentro de las malformaciones arteriovenosas, la agenesia unilateral de una arteria pulmonar es considerada como una de las más raras, con pocos casos descritos en la literatura y disminuyendo su número en casos pediátricos. Se presenta el caso de un lactante femenino con antecedente de tos húmeda y episodios de hemoptisis, lo que lleva a realizar tomografía axial computarizada de alta resolución contrastada y el diagnóstico de agenesia de la arteria pulmonar.
\end{abstract}

Palabras clave: Malformaciones arteriovenosas, hemoptisis, agenesia de arteria pulmonar.

\section{INTRODUCCIÓN}

Dentro de las malformaciones arteriovenosas, la agenesia unilateral de una arteria pulmonar es considerada como una de las más raras, con pocos casos descritos en la literatura y disminuyendo su número en casos pediátricos. Desde su primera descripción por Fraentzel en 1868, esta malformación está casi siempre asociada a malformaciones cardíacas o genéticas y el número de no asociados a ambas situaciones se vuelve realmente escaso, situación por la que se expone el siguiente caso.

\section{PRESENTACIÓN DEL CASO}

Lactante femenino de un año y un mes de edad, producto de la primera gesta, embarazo normoevolutivo, obtenida

\section{Correspondencia:}

\section{Dr. Mario Soto-Ramos}

Universidad Autónoma de Chihuahua, Chihuahua, México.

Correo electrónico: msoto67@gmail.com

Trabajo recibido: 03-VI-2020; aceptado: 10-VII-2020.

Citar como: Soto-Ramos M, Hernández-Saldaña R, Hinojos-Gallardo LC, Mendoza-Enriquez MA, Márquez-Rodríguez YA, Guerrero-Lucio N. Agenesia de rama derecha de la arteria pulmonar. Neumol Cir Torax. 2020;79(3):180184. https://dx.doi.org/10.35366/96654
ABSTRACT. Among arteriovenous malformations, unilateral agenesis of a pulmonary artery (UPAA) is definitely considered one of the rarest, with few cases described in the literature, and its number decreasing in pediatric cases. The case of a female infant with a history of wet cough and episodes of hemoptysis is presented, which leads to performing a contrast-enhanced TACAR and diagnosing agenesis of the pulmonary artery.

Keywords: Arteriovenous malformations, hemoptysis, agenesis of pulmonary artery.

vía abdominal a las 40 semanas de gestación, secundaria a no progresión en el trabajo de parto, con peso de $3.500 \mathrm{~kg}$, talla de $50 \mathrm{~cm}$, Apgar 8-9. Antecedente de una hospitalización previa a los tres meses de edad por una bronquiolitis, estando hospitalizada por una semana. Posterior a esto, la paciente padeció eventos de tos húmeda con episodios de exacerbaciones y remisiones, por lo que es tratada con esteroide inhalado y broncodilatador de acción prolongada, mejorando los síntomas. Sin embargo, a los ocho meses presentó un cuadro de tos húmeda y expectoración con sangre, por lo que es valorada por el Servicio de Otorrinolaringología donde realizan rinoscospia, llegando a la conclusión de que se trataba de epistaxis. Posterior a esto se mantuvo asintomática.

Al año de edad comenzó con un cuadro de tos húmeda, congestión nasal y rinorrea hialina, por lo que acude a valoración donde se da manejo con broncodilatador, sin mejoría de los síntomas. El día previo a su ingreso por este padecimiento, mostró un evento de tos húmeda que se acompañó de salida de sangre fresca por la boca en escasa cantidad; sin embargo, el día de su internamiento comenzó con hemoptisis y en pozos de café, por tanto, se decide su internamiento para su abordaje diagnóstico.

Es valorada por el Servicio de Otorrinolaringología por el evento previo, en donde concluyen que no hay evidencia de sangrado ni en faringe ni en fosas nasales; asimismo, descartan la presencia de hemangiomas. Al ser revisada por 
el Servicio de Gastroenterología concluyen también que es necesario hacer una endoscopia pulmonar y digestiva.

Se realiza panendoscopia, donde se evidencia una esofagitis péptica crónica. En la broncoscopia sólo se observó una traqueobronquitis leve e hipersecreción de moco, se tomó muestra y se obtiene moco de aspecto ligeramente rojizo que es enviado a patología, el área reportó hemosiderófagos y un índice de lipófagos de 83.

Después de dicho procedimiento, la paciente se mantuvo asintomática y en buenas condiciones generales planteando el alta; no obstante, presenta hemoptisis de $30 \mathrm{~mL}$ de sangre fresca, por lo que se difiere el alta y se solicita tomografía axial computarizada de alta resolución (TACAR) y gammagrama con búsqueda de mucosa gástrica ectópica, así como biometría de control, esta última sin un descenso significativo (de $12.5 \mathrm{~g} / \mathrm{dL}$ en su ingreso a $12.2 \mathrm{~g} /$ $\mathrm{dL}$ ). La TACAR simple y contrastada muestra fuga del medio de contraste hacia el lóbulo superior izquierdo, lo que hace sugestivo el diagnóstico de una malformación arteriovenosa pulmonar, también llama la atención la hipoplasia/agenesia de la arteria pulmonar derecha, además de observarse un vaso grande proveniente de la aorta torácica que se dirige hacia el pulmón derecho, considerándose arteria bronquial ingurgitada (Figuras 1 y 2), por lo que se solicita interconsulta a los servicios de Cirugía de Tórax y Hemodinamia.

El cateterismo cardíaco se reportó con los siguientes resultados: AP 33/22 mmHg, VD 29/15 mmHg, AD 5 mmHg, AO 79/50 mmHg. Se aprecia hipoplasia/agenesia de la arteria pulmonar derecha, además de una estenosis leve de la arteria pulmonar izquierda (Figuras 3 a 5). Se comentó el caso con el área de Cirugía de Tórax y Cardiovascular para su posible plastía y se transfunde un concentrado eritrocitario debido a que bajó la $\mathrm{Hb} 2$ gramos con respecto a la biometría de su internamiento. Mediante el cateterismo, se realizó embolización de la arteria bronquial que estaba

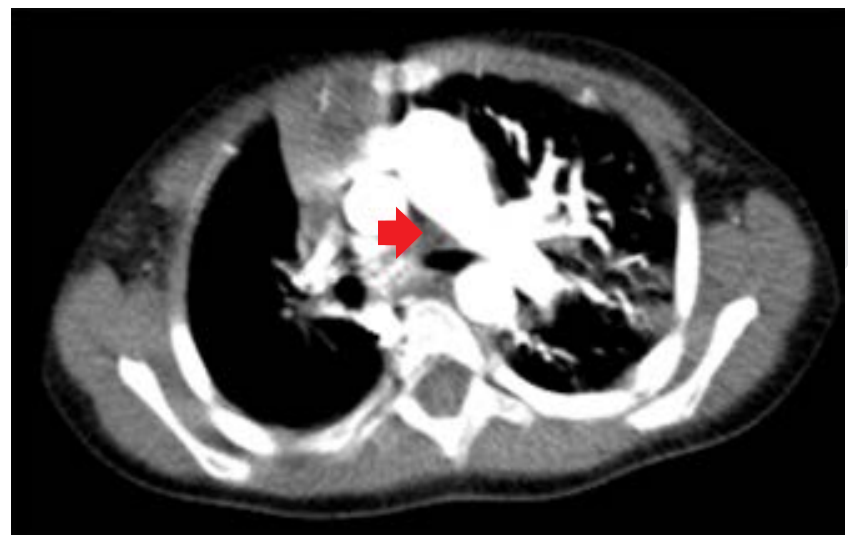

Figura 1: Imagen de TAC con contraste, fase mediastinal, corte axial, en donde se puede apreciar con la flecha roja la ausencia de la rama derecha de la arteria pulmonar.

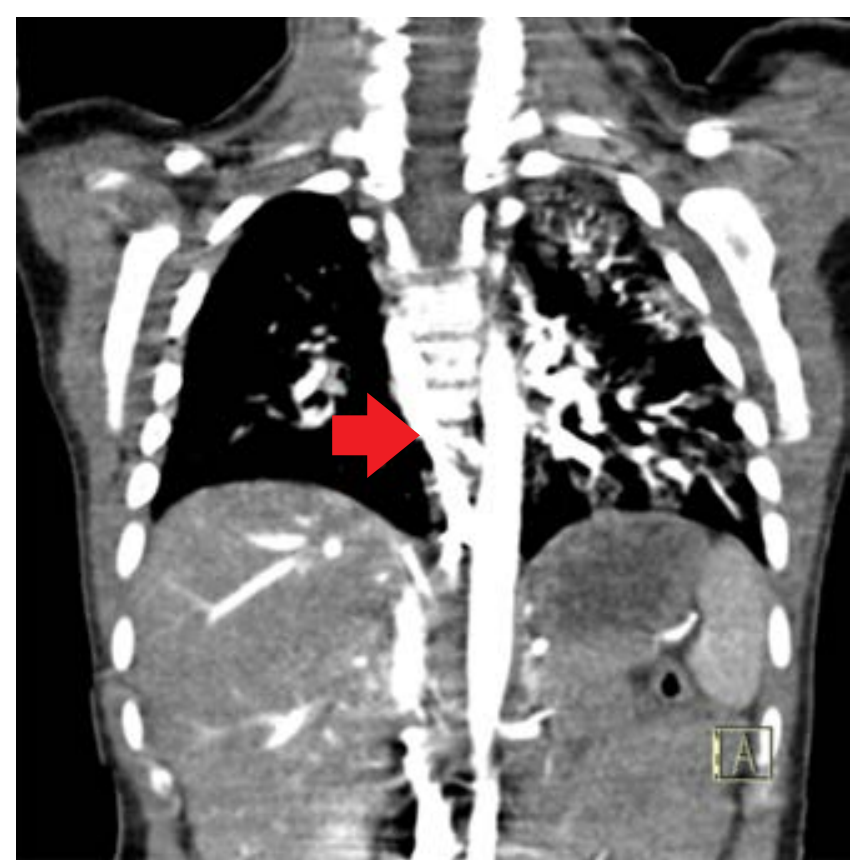

Figura 2: Imagen de TAC contrastada, fase mediastinal, corte coronal, en donde se puede observar una rama nutricia de la aorta descendente hacia el hemitórax derecho (flecha roja).

originando el sangrado y fue necesario realizar una nueva embolización dos semanas después del primer cateterismo a causa de que volvió a presentar hemoptisis, por lo que se contempla realizar entonces neumonectomía total derecha.

Se realiza toracotomía lateral izquierda donde se encontraron múltiples arterias y vasos, surgiendo de mediastino hacia el parénquima pulmonar, se desarrolla doble ligadura de la vena pulmonar inferior con posterior sección distal a la ligadura, se hace sección del bronquio principal derecho, se obstruye por pinzamiento, se asegura ventilación contralateral, se efectúa sección y sutura. Durante el acto quirúrgico no se identificó arteria pulmonar derecha.

\section{DISCUSIÓN}

La agenesia unilateral de una arteria pulmonar (AUAP) fue descrita por primera vez por Fraentzel en 1868, la cual es una malformación congénita rara con pocos reportes en la literatura. ${ }^{1}$ Se desconoce la prevalencia, pero se habla de que entre el $15-30 \%$ cursa de manera asintomática y esos son los casos que se diagnostican en la edad adulta. ${ }^{2}$ Bouros y colaboradores reportaron una prevalencia estimada de 1 : 200,000 individuos ${ }^{3,4}$ con sólo 150 casos reportados desde su primera descripción en 1868. La AUAP es dos veces más común en el lado derecho. Se estima una incidencia de 1 por cada 200,000 a 300,000 recién nacidos vivos, sin predilección por algún género en especial. La mayoría de los 
Neumol Cir Torax. 2020; 79 (3): 180-184

casos se diagnostican en el primer año de vida y son tratados antes de esta edad, la mediana de edad de diagnóstico es a los 14 años, aunque hay varios casos reportados en la edad adulta. ${ }^{5,6}$ Por lo común se asocian con anormalidades cardíacas como la tetralogía de Fallot, persistencia de conducto arterioso y el tronco arterioso común, además se han descrito asociaciones con coartación aórtica y dos más asociados con síndrome de Turner.,78

Como en la literatura, nuestro caso es similar a lo descrito, presentándose del lado derecho la agenesia de la arteria pulmonar aparentemente asintomática. En los casos en que se presenta del lado izquierdo se asocia con cardiopatías, hipertensión pulmonar o insuficiencia cardíaca; por lo común, los que padecen estas manifestaciones suelen diagnosticarse de manera temprana. ${ }^{8}$ Harkel y su equipo encontraron un $7 \%$ de mortalidad global, que los síntomas de hipertensión pulmonar estaban presentes en el $44 \%$, disnea de esfuerzo y tolerancia limitada al ejercicio en $40 \%$, hemoptisis en $20 \%$, infecciones pulmonares recurrentes en el $37 \%{ }^{9}$

Se desconoce con precisión cuál es el origen de tal anormalidad, aunque en el ámbito embriológico se tiene conocimiento de que las arterias pulmonares surgen del sexto arco aórtico, con base en ello se cree que puede haber dos maneras en las que se puede dar el defecto: 1) la reabsorción temprana de la porción proximal del sexto arco aórtico y 2) una septación defectuosa del tronco arterioso. La agenesia de la rama pulmonar derecha o izquierda puede presentarse con ausencia parcial o total del pulmón ipsilateral. En caso de ser parcial, el pulmón se nutre de las

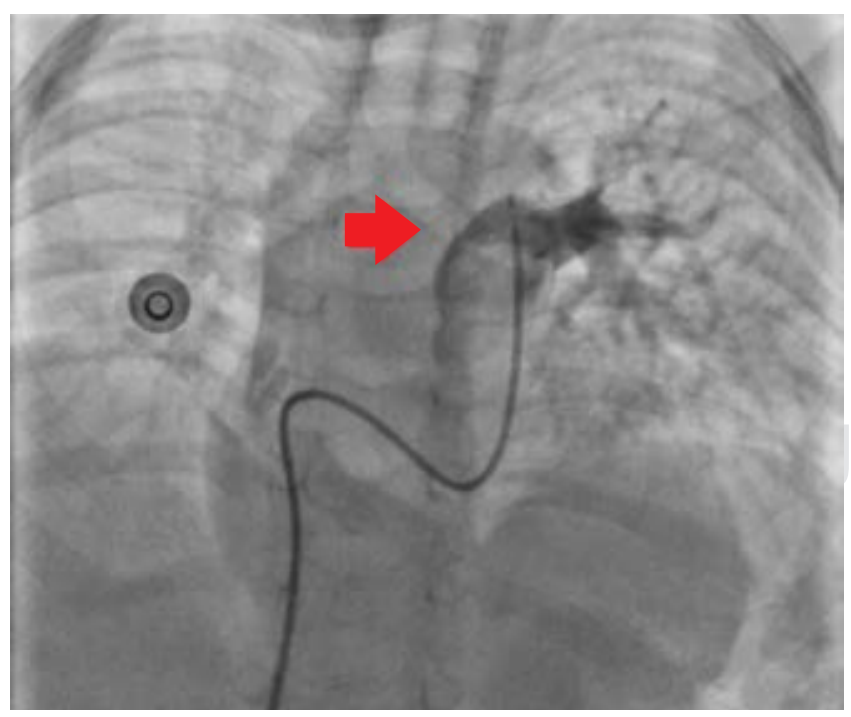

Figura 3: Imagen de cateterismo cardíaco en donde posterior a la administración del medio de contraste se ve la arteriografía de lado izquierdo, no así del lado derecho, evidenciando la ausencia de la rama derecha de arteria pulmonar (flecha roja).

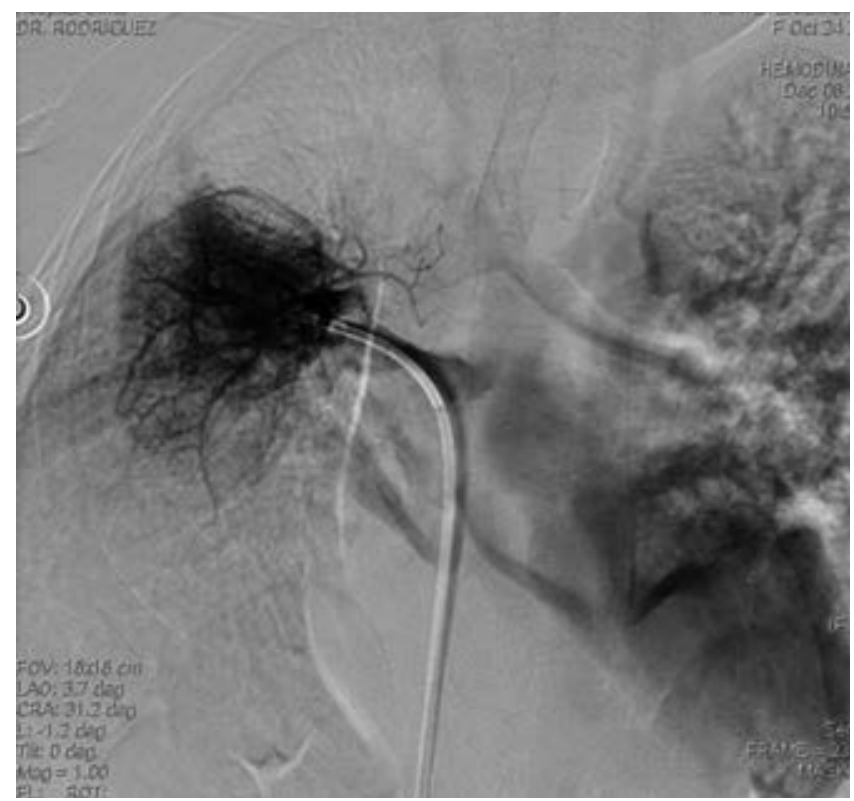

Figura 4: Cateterismo cardíaco en donde se aborda por la arteria pulmonar, encontrando fuga del medio de contraste, sin encontrar un retorno adecuado.

arterias mamarias, intercostales o bronquiales, siendo estas últimas el caso similar a las del caso expuesto. ${ }^{7}$

La sintomatología es variada, como ya mencionamos del $15-30 \%$ pueden ser asintomáticos, pero dentro de las manifestaciones clínicas más frecuentes están la disnea, hemoptisis y la hematemesis, en el caso expuesto en esta investigación se presentaron las dos últimas, coincidiendo así con lo mostrado en la literatura. Dentro de otras manifestaciones se pueden encontrar infecciones respiratorias frecuentes, edema pulmonar hasta en un $12 \%$ y en pacientes mayores la fatiga al ejercicio representó el $40 \%$ de los casos. A la exploración física en los casos asintomáticos es totalmente normal, no así para los que padecen otras patologías, donde a la exploración van a mostrar datos de hipertensión pulmonar preferentemente. Puede haber asimetría torácica si hay hipodesarrollo del lado afectado, disminución de los ruidos respiratorios del mismo lado, con desviación del mediastino hacia el lado afectado. ${ }^{2-8}$ La patogénesis no está clara, pero incluye problemas de aclaramiento mucociliar y disminución de la liberación de células inflamatorias debido a la ausencia de perfusión pulmonar. El resultado es un pulmón inmunodeficiente, que es susceptible incluso a patógenos oportunistas.

Las sibilancias refractarias están mal etiquetadas, ya que el asma grave puede ser una forma inusual de presentación, pues los pacientes presentan broncoconstricción debido a hipocapnia alveolar. La AUAP debe permanecer en el diagnóstico diferencial de sibilancias persistentes a pesar de que ésta no es una presentación habitual de la enfermedad..$^{10}$ 
Para el diagnóstico es siempre importante contar con una radiografía de tórax donde se puede observar un tórax asimétrico, con disminución de los arcos costales y elevación del diafragma del lado afectado, así como el mediastino llevado al lado ipsilateral. El hallazgo más común es la hiperlucencia del lado sano. ${ }^{1-6}$ Aunque el gammagrama ventilatorio-perfusorio permite una adecuada evaluación de dichos aspectos, no permite diferenciar entre una agenesia, una oclusión trombótica o una estenosis de la rama pulmonar. ${ }^{5-7}$ Otra alternativa es el ecocardiograma Doppler color transtorácico y transesofágico, en el que se pueden revelar defectos congénitos o hipertensión pulmonar. ${ }^{6,7}$ La tomografía y la resonancia magnética tienen una relevancia marcada para el diagnóstico en la que la primera por lo común es suficiente para el diagnóstico definitivo, proporciona información detallada y determina la presencia de malformaciones cardiovasculares. ${ }^{5}$ Los hallazgos parenquimatosos incluyen bronquiectasias, patrón en mosaico. ${ }^{6}$ El diagnóstico definitivo se realiza mediante la angiografía, demostrando la ausencia completa de una de las ramas de la arteria pulmonar.

En pacientes con AUAP, todo el gasto cardíaco será desviado al pulmón normal contralateral, lo que puede predisponer a la hipertensión pulmonar a largo plazo con complicaciones asociadas que incluyen dilatación aneurismal de la arteria pulmonar, disección e incluso ruptura. Un metaanálisis por Ten Harkel y colaboradores sugiere que casi la mitad de todos los casos con AUAP aislada desarrollarán hipertensión pulmonar debido a un aumento progresivo en la resistencia vascular pulmonar. La interven-

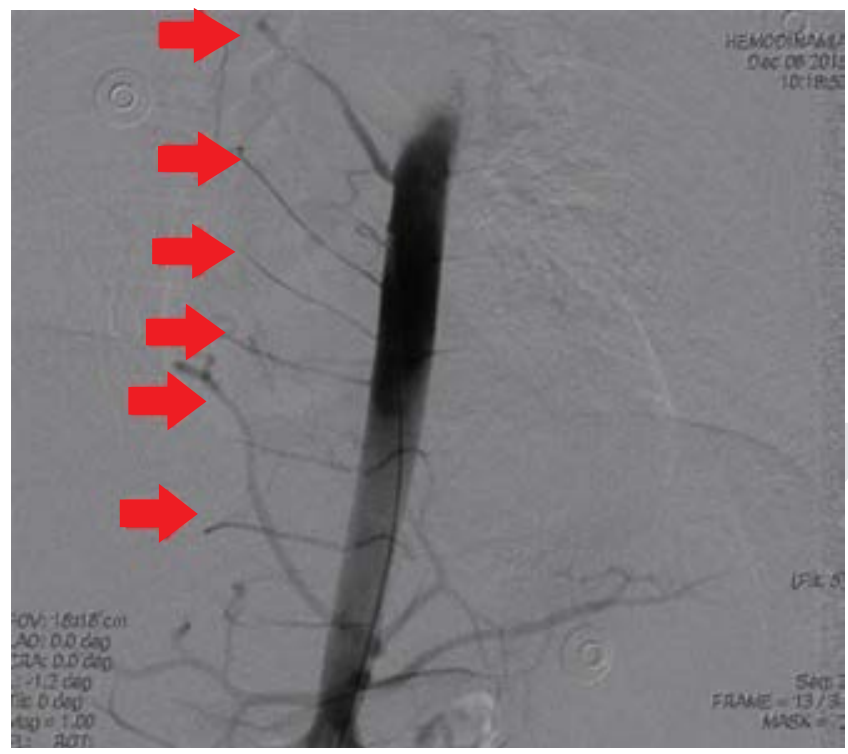

Figura 5: Cateterismo cardíaco, se pueden evidenciar múltiples ramas bronquiales provenientes de la aorta descendente hacia el pulmón derecho (flechas rojas). ción quirúrgica temprana puede dar como resultado una regresión de la hipertensión pulmonar antes de que se vuelva irreversible y así prevenir la falla ventricular derecha. El manejo óptimo requiere un enfoque multidisciplinario que involucre a un cirujano cardiotorácico, médico respiratorio y radiólogo intervencionista. El estándar de oro del tratamiento aún se debate. Todos los autores concuerdan en que se debe adaptar al perfil del paciente, variando desde la revascularización pulmonar en casos pediátricos seleccionados a la embolización endovascular cuando sea posible en adultos, terminando con neumonectomía en pacientes que no responden a terapias previas. ${ }^{8}$

En la literatura, la indicación más común para el tratamiento invasivo es hemoptisis masiva o recurrente, la embolización de la arteria bronquial es menos invasiva y puede llevar menos riesgo de procedimiento que una neumonectomía; sin embargo, las limitaciones incluyen la falta de experiencia, dificultad de la técnica y mayores tasas de recurrencia a largo plazo (hasta 25\%) debido a la extensa colateralización. Además, puede llevar más tiempo hospitalario y compromete la seguridad del paciente en un escenario agudo. La embolización selectiva de arterias bronquiales y arterias sistémico-pulmonares está indicada en caso de detección de una fuente de sangrado activo durante la angiografía selectiva o en pacientes con reserva pulmonar pobre u otras contraindicaciones para la cirugía. Asimismo, repetir la embolización debería ser evitado debido al riesgo teórico de infarto pulmonar o paraplejia.

\section{CONCLUSIONES}

Cuando la hemoptisis es recurrente y masiva, debe considerarse el tratamiento invasivo para evitar situaciones que ponen en peligro la vida. Cuando es factible, la embolización selectiva podría ser efectiva; sin embargo, en manos expertas, la neumonectomía es segura y curativa. La contribución del pulmón afectado al intercambio de gases suele ser mínima, lo que imposibilita un procedimiento de conservación del pulmón. ${ }^{5-10}$ Los médicos deben ser conscientes de la posibilidad de casos no diagnosticados de AUAP que presentan infecciones respiratorias recurrentes. Una radiografía de tórax es generalmente la investigación inicial que sugiere el diagnóstico que puede confirmarse mediante TACAR. La angiografía está reservada para pacientes que requieren embolización o cirugía de revascularización.11

\section{REFERENCIAS}

1. Fraentzel O. Ein Fall von abnormer communication der aorta mit der arteria pulmonalis. Virchow's Arch F Path Anat. 1868;43:420-426.

2. Balci TA, Koç ZP, Kirkil GK, Poyraz AK. Isolated left pulmonary artery agenesis: a case report. Mol Imaging Radionucl Ther. 2012;21(2):8083. Available in: https://doi.org/10.4274/mirt.7 
3. Britton J, Sachithanandan A, Srinivasan L, Ghosh S. Pneumonectomy for congenital isolated unilateral pulmonary artery agenesis. Med $\mathrm{J}$ Malaysia. 2011:66(4):363-364.

4. Sanna S, Dell'Amore A, Monteverde M, Argnani D. Unilateral pulmonary artery agenesia complicated by massive haemoptysis in an adult female. Heart Lung Circ. 2012;21(3):166-168. Available in: https://doi.org/10.1016/j.hlc.2011.10.012

5. Steiropoulos P, Archontogeorgis K, Tzouvelekis A, Ntolios P, Chatzistefanou A, Bouros D. Unilateral pulmonary artery agenesis: A case series. Hippokratia. 2013;17(1):73-76.

6. Aiyappan SK, Ranga U, Veeraiyan S. Incidentally detected isolated unilateral pulmonary artery agenesis. J Clin Diagn Res. 2013;7(4):780781. Available in: https://doi.org/10.7860/jcdr/2013/4570.2911

7. Parra-Bravo J, Acosta-Valdez JL, Zepeda-Sanabria JR, BeiranaPalencia LG, Rodríguez-Hernández L, Estrada-Loza MJ, et al. Agenesia unilateral de una arteria pulmonar y coartación de aorta en un paciente con síndrome de Turner. Rev Mex Cardiol. 2002;13(4):162-170.
8. Adán LV, Jiménez AO, Martín de Vicente C, García IJP. Isolated right pulmonary arteryagenesis. An Pediatr (Barc). 2017;86(1):46-46. Available in: https://doi.org/10.1016/j.anpedi.2016.04.003

9. Patel J, Davis-McDonald K. P139 one lung to live: a case of unilateral pulmonary artery agenesis. Chest. 2017;151(5):A36. Available in: https://doi.org/10.1016/j.chest.2017.04.039

10. Antonescu-Turcu AL, Bhorade S. Refractory wheezing: an unusual presentation of isolated unilateral pulmonary artery agenesis. Chest. 2003;124(Suppl 4):307S. Available in: https://doi.org/10.1378/ chest.124.4_MeetingAbstracts.307S

11. Hafez HM, Neeraj DR, Supat T. A case of unilateral absence of the pulmonary artery in an adult presenting with uncontrolled asthma. Chest. 2009;136(Suppl 4):3S. Available in: https://doi.org/10.1378/ chest.136.4_MeetingAbstracts.3S-d

Conflicto de intereses: Los autores declaran no tener conflicto de intereses. 